\title{
On the Adsorption of Two-State Polymers
}

\author{
N. Yoshinaga \\ Department of Physics, Graduate School of Science, \\ the University of Tokyo, Tokyo 113-0033, Japan. \\ E. Kats \\ Laue-Langevin Institute, F-38042, Grenoble, France, \\ and L. D. Landau Institute for Theoretical Physics, \\ RAS, 117940 GSP-1, Moscow, Russia
}

\author{
A. Halperin* \\ Structures et Propriétés dArchitectures Moléculaires, \\ UMR 5819 (CEA, CNRS, UJF), INAC, \\ CEA-Grenoble, 38054 Grenoble cedex 9, France
}

(Dated: 25/5/08; Received textdate; Revised textdate; Accepted textdate; Published textdate)

\begin{abstract}
Monte Carlo(MC) simulations produce evidence that annealed copolymers incorporating two interconverting monomers, $\mathrm{P}$ and $\mathrm{H}$, adsorb as homopolymers with an effective adsorption energy per monomer, $\epsilon_{e f f}$, that depends on the $\mathrm{PH}$ equilibrium constants in the bulk and at the surface. The cross-over exponent, $\Phi$, is unmodified. The $\mathrm{MC}$ results on the overall $\mathrm{PH}$ ratio, the $\mathrm{PH}$ ratio at the surface and in the bulk as well as the number of adsorbed monomers are in quantitative agreement with this hypothesis and the theoretically derived $\epsilon_{e f f}$. The evidence suggests that the form of surface potential does not affect $\Phi$ but does influence the PH equilibrium.
\end{abstract}




\section{INTRODUCTION}

The interest in polymer adsorption reflects both its practical importance[1] and the associated theoretical challenges[2]. Within this domain the adsorption behavior of neutral water soluble polymers (NWSP), exemplified by polyethylene oxide (PEO) and polyvinyl pyrrolidone (PVP) [3] , occupies a special position. On the applied side this is because of their importance in the formulation of water-based colloidal solutions [4, 5, 6] of practical interest. From a theoretical point of view the adsorption of NWSP poses a distinctive problem: While NWSP such as PEO are homopolymers comprising of chemically identical monomers, they are modeled as "two-state polymers" whose monomers interconvert between hydrophilic (P) and hydrophobic (H) states [7, 8, 9, 10, 11, 12, 13, 14, 15]. These two-state models are invoked in order to rationalize a phase diagram exhibiting a closed insolubility loop, with both upper and lower critical solution temperatures, that is thought to characterize NWSP. The models differ with respect to the precise identification of the two interconverting states. Nevertheless, within all of these models NWSP exhibit an annealed sequence of HP states with a temperature $(\mathrm{T})$ dependent $\mathrm{H} / \mathrm{P}$ ratio. The adsorption behavior of such annealed two-state polymers is expected to differ from that of homopolymers comprising of single monomer species and of quenched random copolymers with a fixed sequence and composition. Thus far, it was investigated using a self consistent field theory [16] of a laterally uniform adsorbed layer, comprising many adsorbed chains, in contact with a polymer solution. In the following we consider, in contrast, the adsorption transition of a single, terminally anchored, two-state polymer and compare it to the corresponding results for homopolymers [2, 17, 18, 19, 20] and for quenched copolymers [21, 22, 23, 24, 25]. In particular, we investigate the adsorption of a non-cooperative two-state polymer using Monte Carlo simulation supplemented by simple theory. Our discussion is mostly concerned with swollen chains under good solvent conditions. Simulation evidence suggests that the adsorption of both homopolymers and of quenched copolymers exhibits identical scaling behavior upon introduction of the appropriate effective adsorption energy per copolymer's monomer, $\epsilon_{\text {eff }}$. The cross-over exponent $\Phi$ characterizing the second-order adsorption transition of these two systems is identical $[21,24,25]$. With this in mind we expect similar behavior for the annealed two-state polymers. Accordingly we first identify the $\epsilon_{e f f}$ for the annealed copolymers case and then analyze the simulation data assuming that such chains adsorb as homopolymers with $\epsilon_{e f f}$ 
specifying the monomer-surface interaction. As we shall see, this picture is consistent with the simulation results. In particular, it allows to collapse the simulation data concerning the number of adsorbed monomers $N_{S}$ onto universal curves and to reproduce the simulation results concerning the total $H$ fraction as well as the $H$ fraction in the bulk and at the surface. Importantly, in distinction to quenched copolymers, the total $H$ fraction as well as the $H$ fraction in the bulk and at the surface are not fixed. These results are of interest from two points of view. First, they complement earlier results on the adsorption transition of homopolymers [2, 17, 18, 19, 20] and quenched copolymers [21, 22, 23, 24, 25]. Second, they provide a starting point for the formulation of a phenomenological free energy description[20] of the adsorption of NWSP modeled as two-state polymers.

In formulating the problem we aimed to capture the generic features common to the various two-state models. These differ with respect to the precise identification of the interconverting states: polar vs apolar [8], hydrated vs nonhydrated [9, 10, 12, 14, 15], or clustered vs nonclustered[11]. With this in mind we focused on the simplest case where the monomers undergo a unimolecular HP interconversion. In the following we confront simple theory with off-lattice Monte Carlo simulations of the adsorption behavior of a single two-state polymer within this minimal model. There is no explicit solvent in the simulation and the monomers states are modeled as Lennard-Jones particles having identical collision diameters. The interactions between of the various monomer-monomer pairs are identical but the interaction parameters with the surface differ with the monomeric state. As a result the $\mathrm{PH}$ interconvesion at the surface and at the bulk are associated with different equilibrium constants (Figure 1). Our model is thus closest to the one proposed by Karlström[8]. $\epsilon_{e f f}$ of the two-state model is obtained in section II using a partition function method similar to that of Moghaddam and Whittington[22] as well as its free energy counterpart. The consequences for the scaling analysis and blob description are discussed in section III. In section IV we present the details of the simulation model and the simulation results are discussed in section $\mathrm{V}$. 


\section{THE EFFECTIVE ADSORPTION ENERGY $\epsilon_{e f f}$ OF ANNEALED COPOLY- MERS}

Two methods can be used to identify $\epsilon_{e f f}$, the adsorption energy of an "effective" monomer at the surface. In one, first used by Moghaddam and Whittington to obtain bounds for quenched copolymers [22, 23], $\epsilon_{e f f}$ is obtained upon recasting the annealed copolymer partition function into a homopolymer-like form containing factors of the form $\exp \left(N_{S} \frac{\epsilon_{e f f}}{k T}\right)$ where $k$ is the Boltzmann constant. Similarly, it is possible to consider the free energy of an annealed copolymer and recognize $\epsilon_{e f f}$ in a term of the form $-N_{S} \epsilon_{e f f}$. These two equivalent methods yield as expected an identical $\epsilon_{\text {eff }}$. We briefly discuss the two versions in order to establish the relationship both to the Moghaddam-Whittington method and to the phenomenological free energy approach.

We begin with the free energy of an adsorbed two-state chain. It comprises two terms, $F_{\text {conf }}$ and $F_{\text {seq. }}$. The first, $F_{\text {conf }}$, allows for the adsorption induced confinement of the chain. It reflects the loss of configurational entropy of the flexible backbone upon confinement to a slab of thickness $D \leqslant R_{F}$ where $R_{F} \sim N^{3 / 5}$ is the Flory radius of the swollen chain. $F_{\text {conf }}$ depends only on $D$, or equivalently $N_{S}$, and its precise functional form is not important for the first part of our discussion. The second term, $F_{\text {seq }}$, accounts for the standard chemical potentials of the $P$ and $H$ monomers in the bulk and at the surface as well as the mixing entropies at the surface and in the bulk. In the good solvent regime the chains are swollen, the monomer concentration is dilute and monomer-monomer interactions have negligible effect on the $P \rightleftarrows H$ equilibrium. The $P H$ sequences in the bulk and at the surface are thus modeled as ideal one-dimensional mixtures and

$$
F_{\mathrm{seq}}\left(N_{S}, x_{H}, x_{S H}\right)=E-T S_{m i x}=N_{B} f_{B}+N_{S} f_{S}=N f_{B}+N_{S}\left(f_{S}-f_{B}\right) .
$$

Here $f_{B}$ and $f_{S}$ are respectively the free energies per monomer in the bulk and at the surface as specified by

$$
\begin{gathered}
f_{S}=x_{S H} \mu_{S H}^{0}+\left(1-x_{S H}\right) \mu_{S P}^{0}+k T\left[x_{S H} \ln x_{S H}+\left(1-x_{S H}\right) \ln \left(1-x_{S H}\right)\right], \\
f_{B}=x_{H} \mu_{H}^{0}+\left(1-x_{H}\right) \mu_{P}^{0}+k T\left[x_{H} \ln x_{H}+\left(1-x_{H}\right) \ln \left(1-x_{H}\right)\right],
\end{gathered}
$$

where the $N$ monomers in the chain comprise $N_{S}$ adsorbed monomers at the surface and $N_{B}=N-N_{S}$ nonadsorbed monomers in the bulk. The $H$ fraction among free monomers is 
$x_{H}=\frac{N_{B H}}{N_{B}}$ while for the adsorbed monomers it is $x_{S H}=\frac{N_{S H}}{N_{S}}$ where $N_{B H}$ and $N_{S H}$ denote respectively the number of $H$ monomers in the bulk and at the surface. The standard chemical potentials of the different species are denoted by $\mu_{i}^{0}$. Note that $f_{B}$ and $f_{S}$ as specified by (21) and (3) are similar to the Zimm-Bragg free energy for the helix-coil transition in the case of zero cooperativity [26]. At this point $f_{B}$ and $f_{S}$ are completely decoupled from each other and from $F_{\text {conf }}$. The equilibrium conditions $\partial f_{B} / \partial x_{H}=0$ and $\partial f_{S} / \partial x_{S H}=0$ lead to the mass action laws in the bulk

$$
\frac{N_{B H}}{N_{B P}}=\frac{x_{H}}{1-x_{H}}=K_{B}=\exp \left(-\frac{\mu_{H}^{0}-\mu_{P}^{0}}{k T}\right)
$$

and at the surface

$$
\frac{N_{S H}}{N_{S P}}=\frac{x_{S H}}{1-x_{S H}}=K_{S}=\exp \left(-\frac{\mu_{S H}^{0}-\mu_{S P}^{0}}{k T}\right) .
$$

Accordingly, a mass action law of the form $K=\frac{x}{1-x}$ or $x=K /(1+K)$ leads, upon substitution in (2) and (3), to the equilibrium free energies per monomer at the surface and in the bulk

$$
\begin{gathered}
f_{S}^{e q}=\mu_{S P}^{0}-k T \ln \left(1+K_{S}\right)=-k T \ln \left[\exp \left(-\frac{\mu_{S P}^{0}}{k T}\right)+\exp \left(-\frac{\mu_{S H}^{0}}{k T}\right)\right], \\
f_{B}^{e q} \equiv \epsilon_{B}=\mu_{P}^{0}-k T \ln \left(1+K_{B}\right)=-k T \ln \left[\exp \left(-\frac{\mu_{P}^{0}}{k T}\right)+\exp \left(-\frac{\mu_{H}^{0}}{k T}\right)\right] .
\end{gathered}
$$

In turn, these yield $F_{\text {seq }}\left(N_{S}, x_{H}, x_{S H}\right)=N f_{B}^{e q}+N_{S}\left(f_{S}^{e q}-f_{B}^{e q}\right) \equiv N \epsilon_{B}-N_{S} \epsilon_{e f f}$ at equilibrium and

$$
\epsilon_{e f f}=f_{B}^{e q}-f_{S}^{e q}=\mu_{P}^{0}-\mu_{S P}^{0}+k T \ln \frac{\left(1+K_{S}\right)}{\left(1+K_{B}\right)}=k T \ln \left[\frac{\exp \left(-\frac{\mu_{S P}^{0}}{k T}\right)+\exp \left(-\frac{\mu_{S H}^{0}}{k T}\right)}{\exp \left(-\frac{\mu_{P}^{0}}{k T}\right)+\exp \left(-\frac{\mu_{H}^{0}}{k T}\right)}\right]
$$

Our sign convention for $\epsilon_{\text {eff }}$ follows the custom in the phenomenological theories of adsorption [20, 27, 28].

Having obtained $\epsilon_{\text {eff }}$ from the free energy of an adsorbed chain we turn to the partition function argument. The partition function of an annealed two-state polymer at a surface is

$$
Z=\sum_{N_{S}} \sum_{N_{S H}=0}^{N_{S H}=N_{S}} \sum_{N_{B H}=0}^{N_{B H}=N_{B}} C_{N}^{+}\left(N_{S}\right)
$$




$$
\begin{gathered}
\times \frac{\left(N-N_{S}\right) !}{N_{B H} !\left(N-N_{S}-N_{B H}\right) !}\left[\exp \left(-\frac{\mu_{H}^{0}}{k T}\right)\right]^{N_{B H}}\left[\exp \left(-\frac{\mu_{P}^{0}}{k T}\right)\right]^{N-N_{S}-N_{B H}} \\
\times \frac{N_{S} !}{N_{S H} !\left(N_{S}-N_{S H}\right) !}\left[\exp \left(-\frac{\mu_{S H}^{0}}{k T}\right)\right]^{N_{S H}}\left[\exp \left(-\frac{\mu_{S P}^{0}}{k T}\right)\right]^{N_{S}-N_{S H}} .
\end{gathered}
$$

Here $C_{N}^{+}\left(N_{S}\right)$ is the number of chain trajectories with $N_{S}$ surface contacts which is assumed to be identical to that of a homopolymer. $N_{S P}=N_{S}-N_{S H}$ is the number of surface monomers in $P$ state and $N_{B P}=N-N_{S}-N_{B H}$ is the corresponding number among bulk monomers. $Z$ as given by (9) counts all possible free and adsorbed PH sequences and assigns to each sequence the appropriate Boltzmann factor. The summations over $N_{B H}$ and $N_{S H}$ yields

$$
\begin{gathered}
Z=\sum_{N_{S}} C_{N}^{+}\left(N_{S}\right)\left[\exp \left(-\frac{\mu_{H}^{0}}{k T}\right)+\exp \left(-\frac{\mu_{P}^{0}}{k T}\right)\right]^{N-N_{S}} \\
\times\left[\exp \left(-\frac{\mu_{S H}^{0}}{k T}\right)+\exp \left(-\frac{\mu_{S P}^{0}}{k T}\right)\right]^{N_{S}} .
\end{gathered}
$$

$Z_{H B}^{0}=\left[\exp \left(-\frac{\mu_{H}^{0}}{k T}\right)+\exp \left(-\frac{\mu_{P}^{0}}{k T}\right)\right]^{N}=\exp \left(-\frac{N \epsilon_{B}}{k T}\right)$ is the HP contribution to the bulk partition function of a two-state chain comprising $N$ monomers. It counts the HP sequences in the absence of a surface or in the presence of a perfectly non-adsorbing surface. We now introduce $\widetilde{C}_{N}^{+}\left(N_{S}\right)=C_{N}^{+}\left(N_{S}\right) \exp \left(-\frac{N \epsilon_{B}}{k T}\right)$ which allows for both the backbone configurations and the HP sequence at a non-adsorbing surface. In turn, this yields a "homopolymer-like" $Z$

$$
Z=\sum_{N_{S}} \widetilde{C}_{N}^{+}\left(N_{S}\right)\left[\frac{\exp \left(-\frac{\mu_{S H}^{0}}{k T}\right)+\exp \left(-\frac{\mu_{S P}^{0}}{k T}\right)}{\exp \left(-\frac{\mu_{H}^{0}}{k T}\right)+\exp \left(-\frac{\mu_{P}^{0}}{k T}\right)}\right]^{N_{S}}=\sum_{N_{S}} \widetilde{C}_{N}^{+}\left(N_{S}\right) \exp \left(N_{S} \frac{\epsilon_{e f f}}{k T}\right)
$$

allowing to identify $\epsilon_{e f f}$, as given by equation (8).

The free energy formulation translates into the partition function description via $F=-k T \ln Z$. In particular, for each set $N_{S}, x_{H}, x_{S H}$ the sequence partition function is approximately $Z_{\text {seq }}\left(N_{S}, x_{H}, x_{S H}\right)=\exp \left[-\frac{F_{s e q}\left(N_{S}, x_{H}, x_{S H}\right)}{k T}\right]$ and $Z_{\text {seq }}=$ $\sum_{N_{S}} \sum_{x_{H}} \sum_{x_{S H}} C_{N}^{+}\left(N_{S}\right) \exp \left[-\frac{F_{\text {seq }}\left(N_{S}, x_{H}, x_{S H}\right)}{k T}\right]$ where $C_{N}^{+}\left(N_{S}\right)$ containing the information on $N_{S}$ corresponds roughly to $\exp \left(-\frac{F_{\text {conf }}}{k T}\right)$. This correspondence is incomplete because it is based on the Stirling approximation and the implicit assumption that $1 \ll N_{S}<N$, $1 \ll N_{B}<N, 1 \ll N_{S H}<N_{S}$ and $1 \ll N_{B H}<N_{B}$. Alternatively, one may begin with the partition function and obtain the free energy formulation upon replacing 
the factorials $x$ ! in $Z_{\text {seq }}$ by $\exp (x \ln x-x)$. As we discussed, $\epsilon_{\text {eff }}$ can be obtained from the minimized free energy which corresponds to the maximal term of $Z$. Minimization of $F$ yields simultaneously $\epsilon_{\text {eff }}$ and the equilibrium constants $K_{B}$ and $K_{S} . K_{B}$ and $K_{S}$ are obtainable from $Z$ upon minimizing of the individual terms subject to the caveats noted earlier. The Moghaddam-Whittington(MW) annealed approximation of the adsorption of quenched copolymers [22] provided the starting point of our discussion of the annealed copolymers adsorption. Within this approximation a quenched copolymer with a fixed average $H$ fraction, $N_{H} / N=p$, and a quenched sequence is modeled as a copolymer with a fixed $p$ but with an annealed sequence [29]. The two treatments differ in two respects: (i) within the MW treatment $f_{B}=0$. (ii) The standard chemical potentials of monomers at the surface, $\mu_{S H}^{0}$ and $\mu_{S P}^{0}$ are replaced in the MW approach by $\tilde{\mu}_{S H}^{0}=\mu_{S H}^{0}-\ln p$ and $\tilde{\mu}_{S P}^{0}=\mu_{S P}^{0}-\ln (1-p)$. Accordingly $F_{\text {seq }}\left(N_{S}, x_{H}, x_{S H}\right)=N_{S} f_{S}$ where $f_{S}=x_{S H} \tilde{\mu}_{S H}^{0}+\left(1-x_{S H}\right) \tilde{\mu}_{S P}^{0}+k T\left[x_{S H} \ln x_{S H}+\left(1-x_{S H}\right) \ln \left(1-x_{S H}\right)\right]$ and the equilib-

rium condition $\partial f_{S} / \partial x_{S H}=0$ leads to the mass action law $\frac{x_{S H}}{1-x_{S H}}=\widetilde{K}_{S}=\frac{p}{1-p} K_{S}$ and to equilibrium $x_{S H}=\frac{p K_{S}}{1-p+p K_{S}}$ instead of the $x_{S H}=\frac{K_{S}}{1+K_{S}}$ as obtained for the fully annealed polymer considered by us. For the particular case considered by MW, where $\mu_{S P}^{0}=0$ and $\mu_{S H}^{0} / k T=-\epsilon_{M W}$, this leads to

$$
\frac{\epsilon_{e f f}}{k T}=-\frac{f_{S}^{e q}}{k T}=\ln \left[\exp \left(-\frac{\widetilde{\mu}_{S P}^{0}}{k T}\right)+\exp \left(-\frac{\widetilde{\mu}_{S H}^{0}}{k T}\right)\right]=\ln \left[p \exp \epsilon_{M W}+1-p\right],
$$

while the partition function (9) is replaced by its MW counterpart

$$
Z=\sum_{N_{S}} \sum_{N_{S H}=0}^{N_{S H}=N_{S}} C_{N}^{+}\left(N_{S}\right) \frac{N_{S} !}{N_{S H} !\left(N_{S}-N_{S H}\right) !} p^{N_{S H}}(1-p)^{N_{S}-N_{S H}} \exp \left(N_{S H} \epsilon_{M W}\right) .
$$

One may thus consider the MW partition function as a special case of equation (10) in which $\mu_{S H}^{0} / k T=-\epsilon_{M W}-\ln p, \mu_{S P}^{0} / k T=\mu_{P}^{0} / k T=-\ln (1-p)$ and $\mu_{H}^{0} / k T=-\ln p$. The two procedures also differ because the MW approach is an approximation when applied to quenched copolymers while its counterpart, as described above, is rigorously applicable to the annealed the two-state polymers considered here [30].

\section{SCALING ANALYSIS AND ADSORPTION BLOBS}

Having identified $\epsilon_{\text {eff }}$ of two-state polymers we are in a position to discuss the scaling analysis of such polymers and the corresponding blob picture. For two-state polymers it 
assumes, as is the case for quenched copolymers, that the polymers adsorb as homopolymers but with an excess adsorption energy per monomer of

$$
\epsilon k T=\epsilon_{e f f}-\epsilon_{e f f}^{c} .
$$

Here $\epsilon_{e f f}^{c}$ is a constant, model dependent, critical adsorption energy at the limit of $\tau=\left(\epsilon_{\text {eff }}-\epsilon_{\text {eff }}^{c}\right) / \epsilon_{\text {eff }}^{c} \rightarrow 0, N \rightarrow \infty$ while $\epsilon N^{\Phi}=$ const $^{\prime}[17]$. In contrast to simple homopolymers where $\epsilon \approx$ const $^{\prime}[20]$ the $T$ dependence of two-state polymers $\epsilon$ is

$$
\epsilon(T)=\ln \frac{\exp \left(-\frac{\mu_{S H}^{0}}{k T}\right)+\exp \left(-\frac{\mu_{S P}^{0}}{k T}\right)}{\exp \left(-\frac{\mu_{H}^{0}}{k T}\right)+\exp \left(-\frac{\mu_{P}^{0}}{k T}\right)}-\frac{\epsilon_{\text {eff }}^{c}}{k T} .
$$

Upon identifying $\epsilon$, the "homopolymer-like" scaling hypothesis for $N_{S}$ is standard. In particular

$$
N_{S} \approx N^{\Phi} g_{s}(x) \text { where } x=\tau N^{\Phi}
$$

where $g_{s}(x)$ is a universal scaling function[17, 18, 20]. At the transition, where $N_{S} \approx N^{\Phi}$, we thus require $g_{s}(x=0) \approx 1$. In the adsorption region, $x>>1, g_{s}(x)$ follows a power law behavior, $x^{q_{s}}$. Since $N_{S} \sim N$ when $x>>1$ this leads to $q_{s}=(1-\Phi) / \Phi$ or $N_{S} \approx N \epsilon^{(1-\Phi) / \Phi}$. Overall, a plot of $N_{S} / N^{\Phi}$ vs $\tau N^{\Phi}$ should collapse the data onto a single curve corresponding to $g_{s}\left(\tau N^{\Phi}\right)$. To this end it is first necessary to determine the model dependent $\epsilon_{\text {eff }}^{c}$. For $\Phi=1 / 2$, the currently accepted value,

$$
\frac{N_{S}^{2}}{N} \sim N^{2 \Phi-1} \sim N^{0}
$$

at the critical point, $\epsilon_{e f f}=\epsilon_{e f f}^{c}$. This indicates that curves of $N_{S}^{2} / N$ vs. $\epsilon_{e f f}$ for different $N$ values intersect at $\epsilon_{e f f}=\epsilon_{\text {eff }}^{c}$ thus allowing to determine $\epsilon_{\text {eff }}^{c}$ from the intersection point[31].

We now return to the free energy per adsorbed chain, $F$. It comprises of two terms $F=F_{\text {conf }}+F_{\text {seq. }}$. The adsorption free energy $F_{\text {seq }} \approx-N_{S} \epsilon k T$ allows for the attractive monomer-surface contacts. $F_{\text {conf }}$ reflects the confinement of the polymer into a slab of thickness $D<R_{F}$. Within the blob picture[20] $F_{\text {conf }} \approx k T N_{b l o b} \approx k T \frac{N}{g}$ where $N_{b l o b}$ is the number of confinement " $D$ blobs" comprising each of $g$ monomers such that $g^{\nu} a \approx D$. Since $N_{S} \approx \frac{N}{g} g^{\Phi}$ we obtain $g \approx\left(\frac{N_{S}}{N}\right)^{\frac{1}{\Phi-1}}$ and thus

$$
\frac{F\left(N_{S}\right)}{k T} \approx N\left(\frac{N_{S}}{N}\right)^{\frac{1}{1-\Phi}}-N_{S} \epsilon
$$


minimization with respect to $N_{S}$ than yields the equilibrium $N_{S}$

$$
N_{S} \approx N \epsilon^{\frac{1-\Phi}{\Phi}}
$$

and the equilibrium free energy of the adsorbed chain

$$
\frac{F_{e q}}{k T} \approx-N \epsilon^{\frac{1}{\Phi}}
$$

In turn, the equilibrium $N_{S}$ together with $x_{H}=K_{B} /\left(1+K_{B}\right)$ and $x_{S H}=K_{S} /\left(1+K_{S}\right)$ determine the total number of $H$ monomers $N_{H}=N_{S} \frac{K_{S}}{1+K_{S}}+\left(N-N_{S}\right) \frac{K_{B}}{1+K_{B}}$ or

$$
\frac{N_{H}}{N} \approx \epsilon^{\frac{1-\Phi}{\Phi}} \frac{K_{S}}{1+K_{S}}+\left(1-\epsilon^{\frac{1-\Phi}{\Phi}}\right) \frac{K_{B}}{1+K_{B}}
$$

As noted earlier, the recent consensus regarding the cross-over exponent suggests $\Phi=1 / 2$ [18]. The corresponding adsorption blobs for three dimensional, self-avoiding chains are specified by

$$
g \approx 1 / \epsilon^{2} ; D \approx a / \epsilon^{6 / 5} ; N_{S} \approx N \epsilon
$$

However, simulation results suggest that finite size effects are important and for finite chains $\Phi \approx 3 / 5$ is more realistic [18]. For relatively short chains the appropriate blob picture may thus involve

$$
g \approx 1 / \epsilon^{5 / 3} ; \quad D \approx a / \epsilon ; N_{S} \approx N \epsilon^{2 / 3}
$$

For the adsorption of free chains, the form of $\epsilon_{e f f}$ leads to distinctive adsorption constant in dilute surface regime, when the adsorbed chains do not overlap. In this regime the chemical potential of the adsorbed chains is $\mu_{a d s} \approx F_{e q}+k T \ln \Gamma$ where $\Gamma$ is the activity of the adsorbed chains and $F_{e q} \approx-k T N \epsilon^{\frac{1}{\Phi}}$ is the standard chemical potential of an adsorbed chain. The chemical potential of the free chains in the bulk is $\mu_{b u l k} \approx k T \ln c_{b u l k}$ where $c_{b u l k}$ is the activity of the free chains. The adsorption isotherm, as obtained from $\mu_{a d s}=\mu_{b u l k}$, is $\Gamma \approx c_{b u l k} K_{a d s}$ where $K_{a d s} \approx \exp \left(N \epsilon^{\frac{1}{\Phi}}\right)$ is the adsorption constant[20]. $K_{a d s}$ for two state polymers and $\Phi=1 / 2$ assumes thus the form

$$
K_{a d s}=\exp N\left\{\ln \frac{\exp \left(-\frac{\mu_{S H}^{0}}{k T}\right)+\exp \left(-\frac{\mu_{S P}^{0}}{k T}\right)}{\left[\exp \left(-\frac{\mu_{H}^{0}}{k T}\right)+\exp \left(-\frac{\mu_{P}^{0}}{k T}\right)\right] \exp \left(\frac{\epsilon_{e f f}^{c}}{k T}\right)}\right\}^{2} .
$$


The $T$ dependence of $K_{a d s}$ as given by (24) differs from that of $K_{a d s}=\exp \left(N \delta^{1 / \Phi}\right)$ as obtained for homopolymer adsorption with $\epsilon=\delta \approx$ const $^{\prime}[20]$.

As we shall see in section IV the approach discussed above accounts well for the simulation results. Confrontation with experimental results is more difficult. The four parameters involved, $\mu_{H}^{0}, \mu_{P}^{0}, \mu_{S P}^{0}$ and $\mu_{S H}^{0}$ are specific both to the particular NWSP considered and to the model used in order to analyze the data. While $\mu_{H}^{0}, \mu_{P}^{0}$ were determined for a number of two-state models, the full set of parameters was only determined for PEO within the Karlström model by fitting phase boundaries and adsorption data[8, 16]. However, while this model is closest to the one we analyze, the two differ in a number of points. For example, in contrast to our model the PP, $\mathrm{HH}$ and $\mathrm{PH}$ interactions within the Karlström model are not identical. With this caveat in mind, this set of $\mu_{H}^{0}, \mu_{P}^{0}, \mu_{S P}^{0}$ and $\mu_{S H}^{0}$ can be used to illustrate the behavior of $\epsilon_{e f f}$ for a "PEO-like" case. In the Karlström model the standard chemical potential of the two-states in the bulk is given by $\mu_{i}^{0}=U_{i}-R T \ln g_{i}$ where $U_{i}$ is the internal energy and $g_{i}$ is a degeneracy factor. The values per mole in KJ are $\mu_{P}^{0}=0$ and $\mu_{H}^{0}=5.086-R T \ln 8[8]$. For the adsorbed species $\mu_{\mathrm{Si}}^{0}=\mu_{i}^{0}-\Delta \epsilon_{i}^{a d}$ where the adsorption energy per mole $\Delta \epsilon_{i}^{a d}$ at methylated silica are $\Delta \epsilon_{P}^{a d}=0.825 \mathrm{KJ}$ and $\Delta \epsilon_{H}^{a d}=1.625 \mathrm{KJ}$ [16]. A rough idea concerning the physical consequences of the Karlström model may be gained upon comparing the $\epsilon_{e f f}$ of a hypothetical P homopolymer, corresponding to PEO modeled as hydrophilic one-state polymer, to the annealed PH homopolymer model of PEO. Such comparison shows that $\epsilon_{e f f}$ of the two-state chain is shifted upwards by roughly a factor of two.

\section{SIMULATION MODEL}

We simulate a two-state polymer terminally anchored to a planar surface. Following Baumgartner [32] the polymer is modeled as freely jointed chain comprising $N$ LennardJones (LJ) particles. The monomers within this bead-spring model interact via a LJ potential

$$
V_{\mathrm{LJ}}=4 \widetilde{\epsilon} \sum_{i, j}\left[\left(\frac{a}{r_{i j}}\right)^{12}-\left(\frac{a}{r_{i j}}\right)^{6}\right],
$$

where $r_{i j}=\left|\mathbf{r}_{\mathbf{i}}-\mathbf{r}_{\mathbf{j}}\right|$ is the distance between monomers $i$ and $j$ such that $|i-j| \geq 2$ and $\mathbf{r}_{i}$ is the position vector of the $i$ th monomer. $\tilde{\epsilon}$ specifies the depth of the potential 
minimum at $r=2^{1 / 6} a$ and $a$, the collision diameter, is the separation for which $V_{\mathrm{LJ}}=0$. This potential exhibits a soft-core steric repulsion at $r \leq a$, and steeply decaying attraction for $r \geq a$. The monomers exist in two interconverting states, $P$ and $H$. It is thus necessary to specify three LJ potentials, corresponding to the interactions between $P P, H H$, and $P H$ monomer pairs, involving altogether six parameters. In the following all three potentials are characterized by the same $a$ and we will thus express all distances in these units. The three remaining parameters, $\widetilde{\epsilon}=\widetilde{\epsilon}_{\mathrm{PP}} k T ; \widetilde{\epsilon}_{\mathrm{PH}} k T ; \widetilde{\epsilon}_{\mathrm{HH}} k T$ determine the strength of interactions between the $P P, P H$, and $H H$ monomers. For the simulation of self avoiding chains we set $\widetilde{\epsilon}_{\mathrm{PH}}=\widetilde{\epsilon}_{\mathrm{HH}}=\widetilde{\epsilon}_{\mathrm{PP}}=0.20$ so that the monomer-monomer interactions are dominated by the excluded volume and contain effectively no attractive contribution. For simulations of ideal polymers $\widetilde{\epsilon}_{\mathrm{PH}}=\widetilde{\epsilon}_{\mathrm{HH}}=\widetilde{\epsilon}_{\mathrm{PP}}=0$. In addition, the bulk $H P$ states are characterized by standard chemical potentials $\mu_{H}^{0}=k T \Delta \epsilon$ and $\mu_{P}^{0}=0$ with $\Delta \epsilon>0$. Accordingly $\Delta \mu^{0}=\Delta \epsilon k T$ specifies the difference in standard chemical potential $\Delta \mu^{0}=\mu_{H}^{0}-\mu_{P}^{0}$ between noninteracting $H$ and $P$ monomers. All monomer pairs, except nearest-neighbors, interact via LJ potentials. Nearest-neighbor monomers along the chain are constrained to $0.7 \leq$ $\left(\mathbf{r}_{i+1}-\mathbf{r}_{i}\right)^{2} \leq 2.0$. Separations outside this range incur an infinite energy penalty thus ensuring connectivity.

We have used two monomer-surface interaction potentials, $U_{\text {wall }}$, both specified in terms of $z$, the distance between the monomer and the surface. One is the contact potential

$$
U_{\text {wall }}= \begin{cases}\infty & z \leq 0 \\ -k T \Delta \epsilon_{i}^{a d} & 0<z \leq 1 \\ 0 & z>1\end{cases}
$$

where $\Delta \epsilon_{i}^{a d}>0$ is the depth of the square well adjacent to the surface as experienced by species $i=P, H$. We will mostly focus on the contact potential because it allows for unambiguous definition of adsorption i.e., a monomer having its center within the slab $0<z \leq 1.0$ is adsorbed. The second, more physical, "10-4" wall potential

$$
U_{\text {wall }}=k T \Delta \epsilon_{i}^{a d}\left[\left(\frac{1}{z}\right)^{10}-2.5\left(\frac{1}{z}\right)^{4}\right],
$$

as obtained by integrating the LJ potentials between the top monolayer atoms of the substrate and a monomer at $z$. Here again $\Delta \epsilon_{i}^{a d}>0$ is a tuning parameter characterizing the strength of the attraction[18]. When using the 10-4 potential the criterion for adsorption 
is somewhat arbitrary and we define monomers with $z<2$ as adsorbed[18]. Note however that monomers within the adsorption slab experience a $z$ dependent $U_{\text {wall }}$. In both cases the values of $\Delta \epsilon_{i}^{a d}$ for the $\mathrm{P}$ and $\mathrm{H}$ states, $\Delta \epsilon_{H}^{a d}$ or $\Delta \epsilon_{P}^{a d}$, differ favoring the surface $\mathrm{H}$ state i.e., $\Delta \epsilon_{P}^{a d}<\Delta \epsilon_{H}^{a d}$. For the contact surface potentials we thus have

$$
\mu_{S H}^{0} / k T=\mu_{H}^{0}-\Delta \epsilon_{H}^{a d}=\Delta \epsilon-\Delta \epsilon_{H}^{a d} \text { and } \mu_{S P}^{0} / k T=\mu_{P}^{0}-\Delta \epsilon_{P}^{a d}=-\Delta \epsilon_{P}^{a d} .
$$

For the 10-4 potentials $\mu_{S H}^{0}$ and $\mu_{S P}^{0}$ are $z$ dependent. However, due to the fast decay of the potential $\mu_{S H}^{0}$ and $\mu_{S P}^{0}$ as given by (28) provide, as we shall see, a reasonable approximation.

The simulations involves chains comprising $N=64,128,256$ monomers. At each Monte Carlo step (MCs) we shift the position of every monomer in the chain and update its HP state using the Metropolis algorithm. This procedure is thus repeated $N$ times per MCs. For each set of parameters the simulation involves $2 \times 10^{7}$ MCs. The system is equilibrated during million MCs. The remaining $1.9 \times 10^{7} \mathrm{MCs}$ are grouped into sets of $10^{4} \mathrm{MCs}$ whose configurational and PH characteristics are averaged for analysis. In a conformational update of the monomer position, a monomer is chosen randomly, its position is shifted by a sufficiently small distance and the resulting energy difference $k T \Delta E$, accounting for LJ interactions, is calculated. When $\Delta E \leq 0$, the operation is accepted and we proceed to the next monomer movement. On the other hand when $\Delta E>0$, the operation is accepted with the probability $\exp (-\Delta E)$. The $\mathrm{PH}$ interconversion updates are implemented in two stages corresponding to the bulk equilibration and its modification by the surface potential. In the "bulk stage" a monomer is randomly chosen and its state is updated with the probability

$$
\left\{\begin{array}{l}
p(\mathrm{P} \rightarrow \mathrm{H})=\exp (-\Delta \epsilon) \\
p(\mathrm{H} \rightarrow \mathrm{P})=1
\end{array}\right.
$$

where $p(\mathrm{P} \rightarrow \mathrm{H})$ and $p(\mathrm{H} \rightarrow \mathrm{P})$ denote respectively the transition probabilities from $\mathrm{P}$ to $\mathrm{H}$, and from $\mathrm{H}$ to $\mathrm{P}$. The detailed balance condition for the bulk equilibrium is $p(\mathrm{P} \rightarrow \mathrm{H}) N_{B P}=$ $p(\mathrm{H} \rightarrow \mathrm{P}) N_{B H} \quad$ yields

$$
\frac{N_{B H}}{N_{B P}}=\frac{x_{H}}{1-x_{H}}=\exp (-\Delta \epsilon) \equiv K_{B}
$$

This procedure is the counterpart of the trial motion in the conformational steps. The PH states are subsequently updated, in $z$ dependent fashion, allowing for the effect of the surface potential on the PH equilibrium. For the contact potential, monomers with $z=1$ are converted following the transition probabilities 


$$
\left\{\begin{array}{l}
P(S \mathrm{P} \rightarrow \mathrm{SH})=\exp \left(\Delta \epsilon_{P}^{a d}-\Delta \epsilon_{H}^{a d}\right) \\
P(S \mathrm{H} \rightarrow \mathrm{SP})=1
\end{array}\right.
$$

where $P(S \mathrm{H} \rightarrow \mathrm{SP})=1$ because $\Delta \epsilon_{P}^{a d}<\Delta \epsilon_{H}^{a d}$. The corresponding detailed balance condition for equilibrium at the surface, $p(\mathrm{P} \rightarrow \mathrm{H}) P(S P \rightarrow S H) N_{S P}=p(\mathrm{H} \rightarrow \mathrm{P}) P(S H \rightarrow S P) N_{S H}$ yields

$$
\frac{N_{S H}}{N_{S P}}=\frac{x_{S H}}{1-x_{S H}}=\exp \left(\Delta \epsilon_{H}^{a d}-\Delta \epsilon-\Delta \epsilon_{P}^{a d}\right)=K_{S} .
$$

In contrast, monomers at $z>1$ experience no surface effect and $K_{B}$ is accordingly unmodified. Similar procedure is used for the 10-4 wall potential with the distinction that the $z$ dependent surface transition probabilities occur, in principle at all $z$ values with subsequent effect on $K_{B}$ as well as on $K_{S}$. However, since the 10-4 potential decays fast the effect on the bulk equilibrium is negligible.

\section{SIMULATION RESULTS}

As opposed to the case of quenched copolymers, whose composition is fixed, the $H$ fraction of our model two-state polymers depends on three tuning parameters $\Delta \epsilon, \Delta \epsilon_{H}^{a d}$ and $\Delta \epsilon_{P}^{a d}$. In the following $\Delta \epsilon_{P}^{a d}$ is fixed while $\Delta \epsilon$ and $\Delta \epsilon_{H}^{a d}$ are varied. Our results are mostly concerned with chains exhibiting self avoiding random walk (SAW) statistics and interacting with the surface via contact potentials. In addition we will comment briefly on the behavior of ideal chains exhibiting random walk (RW) statistics and the effect of 10-4 monomer-surface potentials. Importantly, variation of $\Delta \epsilon_{H}^{a d}$ affects $N_{S H}$ (Figure 2), $N_{H}$ (Figure 3 ) and $N_{S}$ (Figure 4$)$. As seen from Fig $2, N_{S H} / N_{S}=K_{S} /\left(1+K_{S}\right)$ and $N_{B H} / N_{B}=K_{B} /\left(1+K_{B}\right)$ in agreement with equations (4) and (5). To proceed further it is first necessary to determine $\epsilon_{\text {eff }}^{c}$. As noted earlier, curves of $N_{S}^{2} / N$ vs. $\epsilon_{e f f}$ for different $N$ values intersect at $\epsilon_{\text {eff }}=\epsilon_{\text {eff }}^{c}$ provided that $\Phi=1 / 2$. Alternatively, curves of $N_{S}^{2} / N$ vs. $\Delta \epsilon_{H}^{a d}$ will intersect at $\Delta \epsilon_{H}^{c}$ for families of curves of identical $\Delta \epsilon, \Delta \epsilon_{P}^{a d}$, but different $N$ values. Within the accuracy of our data the curves do intersect at a single point (Figure 5) thus lending support to the assumption that the cross-over exponent $\Phi=1 / 2$ describes the

adsorption behavior of two-state polymers. Furthermore, $\epsilon_{e f f}^{c}$ as obtained by substituting $\Delta \epsilon_{H}^{c}$ and the corresponding $\Delta \epsilon, \Delta \epsilon_{P}^{a d}$ values into (8) is a constant (Figure 6) as expected 
within the picture of "homopolymer-like" adsorption. Having obtained $\epsilon_{\text {eff }}^{c}$ we find that the $N_{S}$ vs $\Delta \epsilon_{H}^{a d}$ data collapses onto a universal curve (Figure 7) upon plotting $N_{S} / N^{1 / 2}$ vs $\tau N^{1 / 2}$ where

$$
\tau=\frac{k T}{\epsilon_{e f f}^{c}} \ln \frac{\exp \left(\Delta \epsilon_{H}^{a d}-\Delta \epsilon\right)+\exp \left(\Delta \epsilon_{P}^{a d}\right)}{1+\exp (-\Delta \epsilon)}-1 .
$$

Furthermore, the $N_{H} / N$ data agrees with equation (21) as adapted to the simulation model (Figure 3)

$$
\frac{N_{H}}{N} \approx \epsilon \frac{\exp \left(\Delta \epsilon+\Delta \epsilon_{P}^{a d}-\Delta \epsilon_{H}^{a d}\right)}{\exp \left(\Delta \epsilon+\Delta \epsilon_{P}^{a d}-\Delta \epsilon_{H}^{a d}\right)+1}+(1-\epsilon) \frac{\exp (\Delta \epsilon)}{\exp (\Delta \epsilon)+1} .
$$

The above results concern SAW and contact potentials. The $\epsilon_{\text {eff }}^{c}$ values for RW are lower than those of SAW chains and depend on the wall potential (Figure 6). However, since $\Phi=1 / 2$ applies to SAW as well as RW chains, the $N_{S} / N^{1 / 2}$ vs $\tau N^{1 / 2}$ scaling with the proper choice of $\tau$ is obtained in both cases for adsorption due to contact potentials (Figure $7-8)$.

The adsorption behavior of homopolymers experiencing 10-4 potential and contact potential is indistinguishable[18]. Our discussion suggests that the adsorption of an annealed copolymer is analogous to the adsorption of a homopolymer with $\epsilon_{\text {eff }}$ given by (8). Accordingly we expect the adsorption of annealed homopolymers to be insensitive to the choice of the potential and of the $z$ values used to define adsorbed monomers. Within the accuracy of our simulation, this is indeed the case for the adsorption of RW annealed copolymers where the data is collapsed by plotting $N_{S} / N^{1 / 2}$ vs $\tau N^{1 / 2}$ (Figure 9). It is however important to note that for this case the $P H$ equilibrium is $z$ dependent. Accordingly $N_{S H} / N_{S}=K_{S} /\left(1+K_{S}\right)$ and $N_{B H} / N_{B}=K_{B} /\left(1+K_{B}\right)$ as specified by (30) and (32) only capture the qualitative features of the $\mathrm{PH}$ equilibrium but do not yield quantitative agreement because $\mu_{S H}^{0}$ and $\mu_{S P}^{0}$ as approximated via (28) do not allow for the $z$ dependence of $U_{\text {wall }}$. A quantitative agreement is achieved upon dividing $\Delta \epsilon_{H}^{a d}$ or $\Delta \epsilon_{P}^{a d}$ by 1.5 allowing presumably for the average $U_{\text {wall }}$ experienced within the $z<2$ region (Figure 10).

\section{DISCUSSION}

The adsorption behavior of annealed copolymers may conceivably differ with the details of the model. Our discussion concerned the particular case of non-cooperative two-state 
polymers involving unimolecular $\mathrm{PH}$ interconversion. For this case, our results suggest that the annealed copolymers adsorb as homopolymers once the appropriate effective adsorption energy per monomer at the surface, $\epsilon_{e f f}$, is identified. This $\epsilon_{e f f}$ accounts for the average adsorption energy as well as the mixing entropy of the surface PH monomers at equilibrium. The adsorption of two-state polymers is described by the cross-over exponent $\Phi$ of homopolymer adsorption. In this respect, their behavior is identical to that of quenched copolymers. However, as opposed to quenched copolymers, where $\epsilon_{\text {eff }}$ as obtained from the MW annealed approximation is determined by the fixed average $H / P$ fraction[22, 25], $\epsilon_{e f f}$ in the annealed case depends on the $P H$ equilibrium constants in the bulk and at the surface, $K_{B}$ and $K_{S}$. In other words $\epsilon_{e f f}$ varies with the $H / P$ ratio which, in contrast to the quenched case, depends on the temperature, the adsorption energies and the bulk standard chemical potentials. This picture allows to calculate the number of adsorbed monomers $N_{S}$, the $H / P$ ratio in the bulk and at the surface as well as the overall $H / P$ ratio. The calculated values are in good agreement with the simulation results concerning SAW and RW experiencing contact potentials. Simulation of homopolymer chains suggest that the form of the wall-monomer potential does not affect $\Phi$ and the scaling behavior of $N_{S}$. Since annealed copolymers adsorb as homopolymers characterized by $\epsilon_{\text {eff }}$ one expects similar insensitivity to the details of the potential. In contrast, the $H / P$ ratio is sensitive to the range of the wall potential. Both features are apparent from our results concerning the adsorption of RW annealed copolymers subject to a 10-4 wall potential. As noted earlier, our analysis utilizes a simplified version of the Karlström model. In contrast to this model all monomer-monomer interactions are identical. New features may well occur upon introducing attraction between hydrophobic $\mathrm{H}$ monomers. In the case of free chain in the bulk such interaction can lead to phase separation[8] and to polymer collapse[33]. The preferential adsorption of $\mathrm{H}$ monomers together with $\mathrm{HH}$ attraction may lead to adsorption induced collapse.

Our discussion thus far focused on the comparison between simulation results of annealed and quenched copolymers. The comparison of the experimental situation brings up a second issue. The experimental realization of quenched copolymers is clear and studying the adsorption of the corresponding homopolymers allows to deduce the adsorption energies of the different monomers involved. The situation is more difficult in the case of annealed copolymer as encountered in the modeling of NWSP. The corresponding one state homopolymers do not exist. Furthermore, direct experimental evidence concerning the molecular identity 
of the interconverting states is yet to emerge. As a result the interaction parameters are obtained indirectly by model dependent fitting of experimental data. With this in mind it is important to recall the experimental evidence for two-state models. Two observations are of special interest. One is their ability to predict the qualitative features of the phase diagram and fit experimentally observed phase boundaries [7, 8, 9, 10, 12, 13, 14, 15]. The second concerns the stretching of PEO chains in atomic force microscopy experiments. This reveals that different force laws characterize the strong stretching regime in water and in hexadecane. In particular, the chain extension in water exhibits a plateau characteristic of two-state polymers [34].

Acknowledgement 1 N.Y. benefited from fellowship No. 7662 from the Japan Society for the Promotion of Science (JSPS) as well as from the hospitality of the theory group of the ILL. Part of the numerical calculations in this work was carried out on Altix3700 BX2 at YITP in Kyoto University.

[1] Napper, D. H. Polymeric Stabilization of Colloidal Dispersions; Academic Press: London, 1983.

[2] Eisenriegler, E. Polymers Near Surfaces: Conformation Properties and Relation to Critical Phenomena; World Scientific Publishing Company 1993

[3] Molyneux, P. Ed. Water-Soluble Synthetic Polymers: Properties and Behavior; Volume I \&II CRC Press 1984

[4] Glass J.E. Ed, Water-Soluble Polymers: Beauty with Performance; Oxford University Press, USA 1986

[5] Glass, J.E. Ed., Hydrophilic Polymers: Performance with Environmental Acceptance; Oxford University Press 1996

[6] Williams, P. Ed. Handbook of Industrial Water Soluble Polymers; Wiley-Blackwell NY, 2007

[7] Goldstein, R. J. Chem. Phys. 1984, 80, 5340-5341.

[8] Karlström, G. J. Phys. Chem. 1985, 89, 4962-4964.

[9] Matsuyama, A.; Tanaka, F. Phys. Rev. Lett. 1990, 65, 341-344.

[10] Veytsman, B. J. Phys. Chem. 1990, 94, 8499-8500. 
[11] de Gennes, P.G. CR l'Academie. Sci., Ser. II 1991 313, 1117-1122.

[12] Bekiranov, S.; Bruinsma, R.; Pincus, P. Europhys. Lett. 1993, 24, 183-188.

[13] Jeppesen, C.; Kremer, K. Eurphys. Lett. 1996, 34, 563-568.

[14] Bekiranov, S.; Bruinsma, R.; Pincus, P. Phys. Rev. E 1997, 55, 577-585.

[15] Dormidontova, E. Macromolecules 2002, 35, 987-1001.

[16] Linse P; Björling M. Macromolecules 1991, 24, 6700-6711

[17] Eisenriegler, E.; Kremer, K.; Binder, K.; J. Chem. Phys. 1982, 77, 6296

[18] Metzger, S.; Müller, M.; Binder, K.; Baschnagel, J. Macromol. Theory Simul. 2002, 11, 985995

[19] Luo MB J. Chem. Phys. 2008, 128, 044912

[20] Bouchaud, E; Daoud, M J. Phys. (Orsay) 1987, 37, 1991-2000,

[21] Sumithra K; Baumgaertner, A. J. Chem. Phys 1999, 10, 2727-2731

[22] Moghaddam, MS; Whittington SG J. Phys. A: Math. Gen, 2002, 35, 33-42

[23] Soteros, CE ; Whittington SG J. Phys. A: Math. Gen, 2004, 37, R279-R325

[24] Ziebarth, J D.; Wang, Y; Polotsky,A; Luo M. Macromolecules 2007, 40, 3498-3504

[25] Bhattacharya, S.; Hsu,H.-P.; Milchev, A.;Rostiashvili,V. G.; Vilgis, T. A. Macromolecules 2008, 41, 2920-2930

[26] Grosberg, A. Yu.; Khokhlov, A. R. Statistical Physics of Macromolecules; American Institute of Physics, NewYork, 1994.

[27] de Gennes, P.-G. Scaling Concepts in Polymer Physics; Cornell, University Press: Ithaca, NY, 1979.

[28] Rubinstein, M.; Colby, R. H. Polymer Physics; Oxford University Press: Oxford, U.K., 2003.

[29] A fixed overall $H$ fraction is not gauranteed in the MW annealed model especially for large $\epsilon$ [23].

[30] Our discussion focused on the MW annealed approximation because of its simplicity and proven utility in the analysis of simulation data [24, 25]. We should add that the more general Morita approximation [23] is also obtainable from equation (10) upon choosing $\mu_{P}^{0} / k T=$ $\mu_{S P}^{0} / k T=\lambda p-\ln (1-p),-\mu_{H}^{0} / k T=\lambda(1-p)+\ln p$ and $-\mu_{S H}^{0} / k T=\epsilon_{M W}-\ln p, \mu_{S H}^{0} / k T=$ $\epsilon_{M W}+\lambda(1-p)+\ln p$.

[31] Hsu, H.-P., Nadler, W.; Grassberger P J. Phys. A: Math. Gen, 2005, 38, 775-806

[32] Baumgärtner, A. J. Chem. Phys. 1980, 72, 871-879. 
[33] Yoshinaga, N. ; Bicout, D.J.; Kats E.I.; Halperin, A. Macromolecules 2007, 40, 2201

[34] Oesterhelt, F., Rief M., Gaub, H., New J. Phys. 1999, 1, 6. 

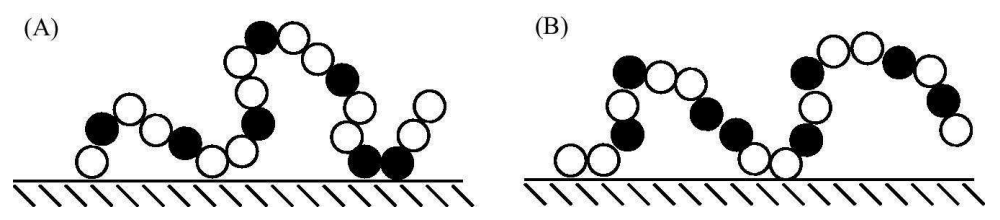

FIG. 1: Two configuration of an adsorbed two-state polymer undergoing $\mathrm{P}$ (full circles) H(empty circles) interconversion in the bulk and at the surface.

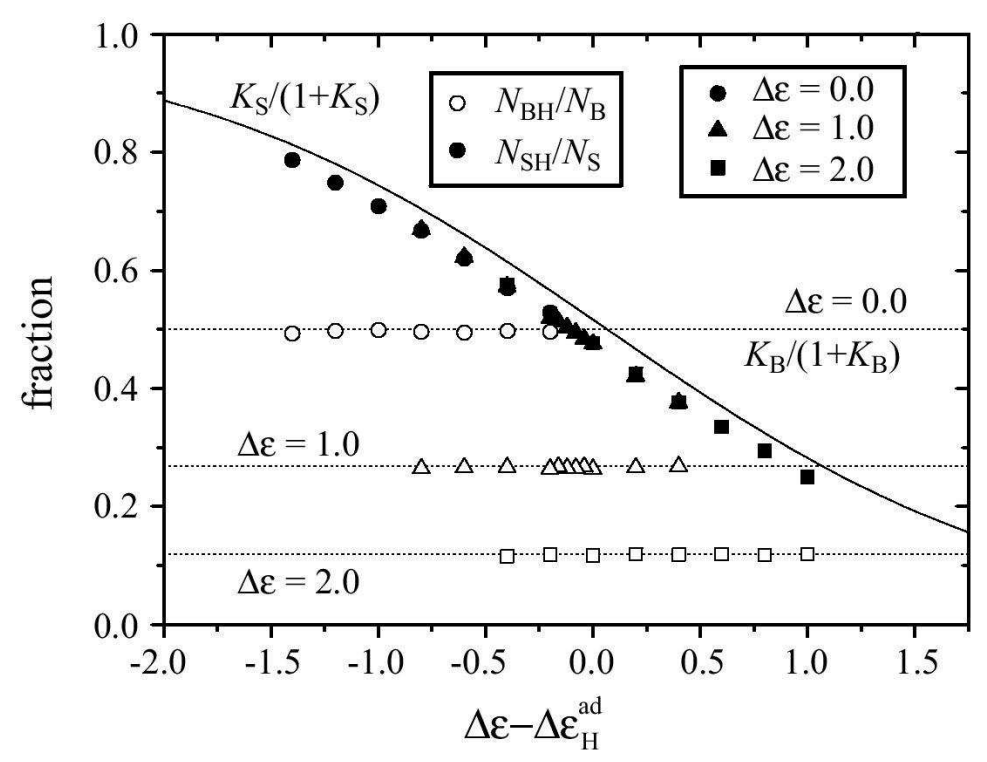

FIG. 2: $\quad N_{B H} / N_{B}$ and $N_{S H} / N_{S}$ as a function of a contact potential $\Delta \epsilon-\Delta \epsilon_{H}^{a d}$ for various $\mu_{H}^{0}-\mu_{P}^{0}=$ $k T \Delta \epsilon$ and the corresponding curves given by (30) and (32). 


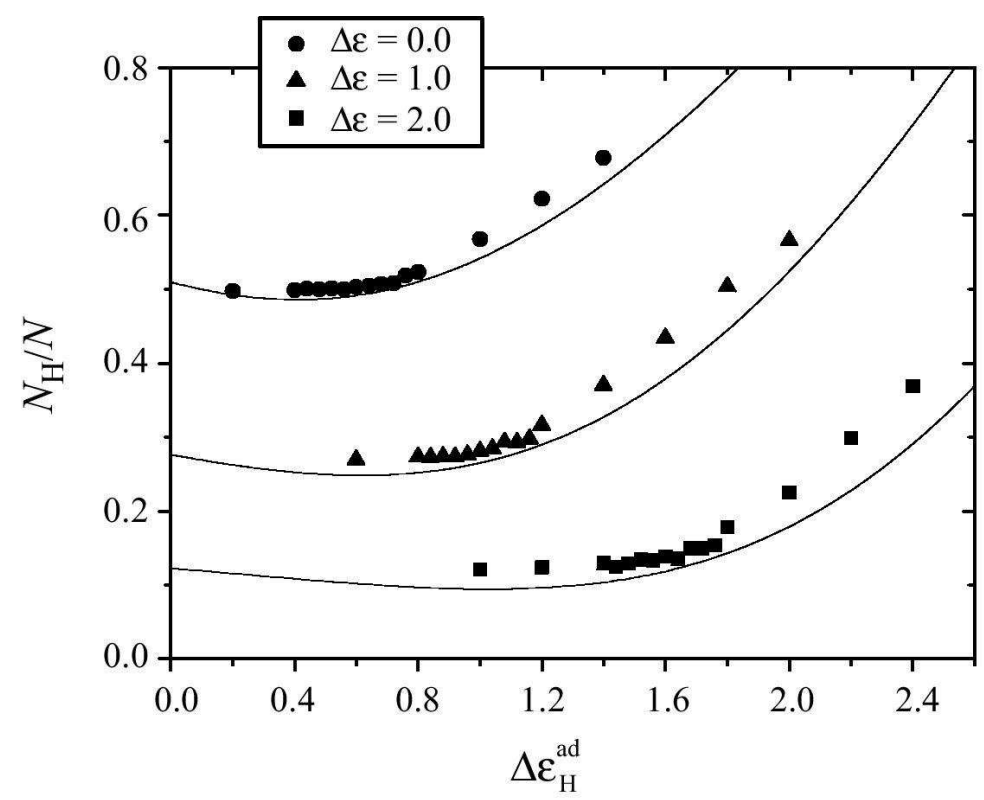

FIG. 3: Plot of $N_{H} / N$ as a function of of a contact potential $\Delta \epsilon_{H}^{a d}$ for various $\mu_{H}^{0}-\mu_{P}^{0}=k T \Delta \epsilon$ and the corresponding curves given by (34).

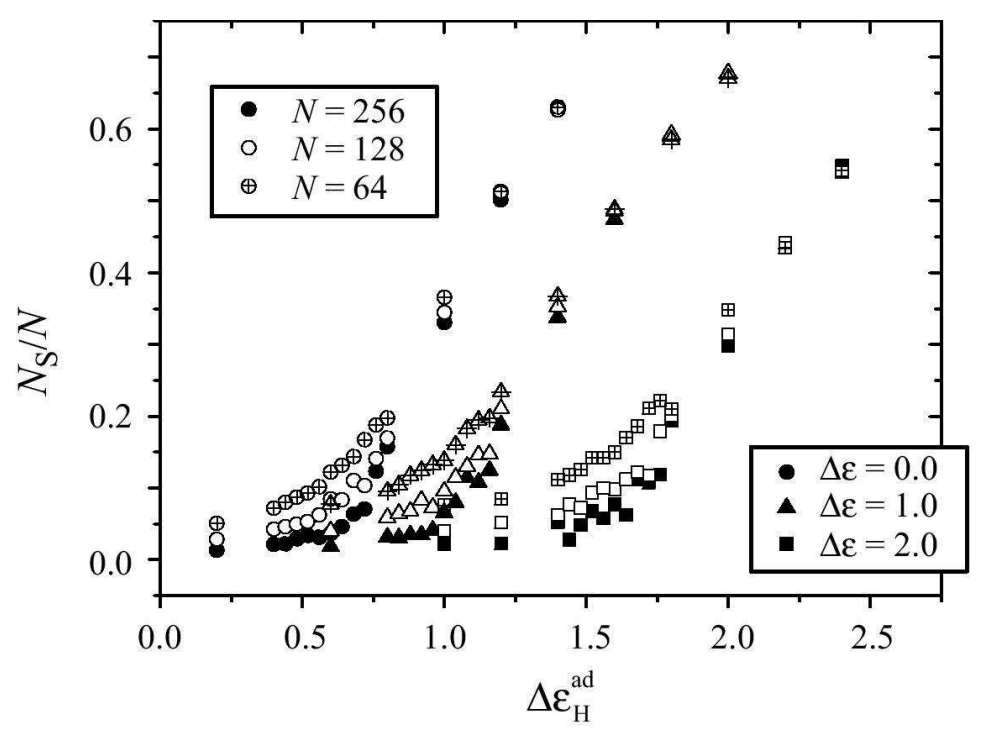

FIG. 4: $N_{S} / N$ vs $\Delta \epsilon_{H}^{a d}$ of a contact potential for self avoiding chains with various $\mu_{H}^{0}-\mu_{P}^{0}=k T \Delta \epsilon$ and $N$. 


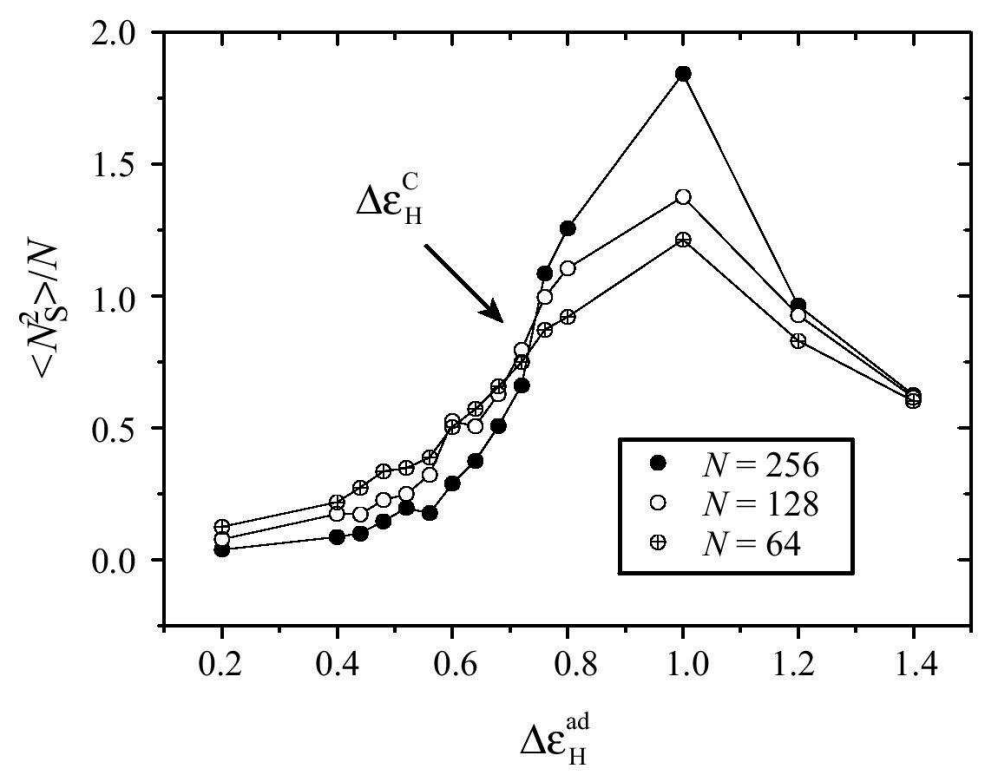

FIG. 5: $\quad N_{S}^{2} / N$ vs $\Delta \epsilon_{H}^{a d}$ for various $N$ and $\Delta \epsilon=0$.

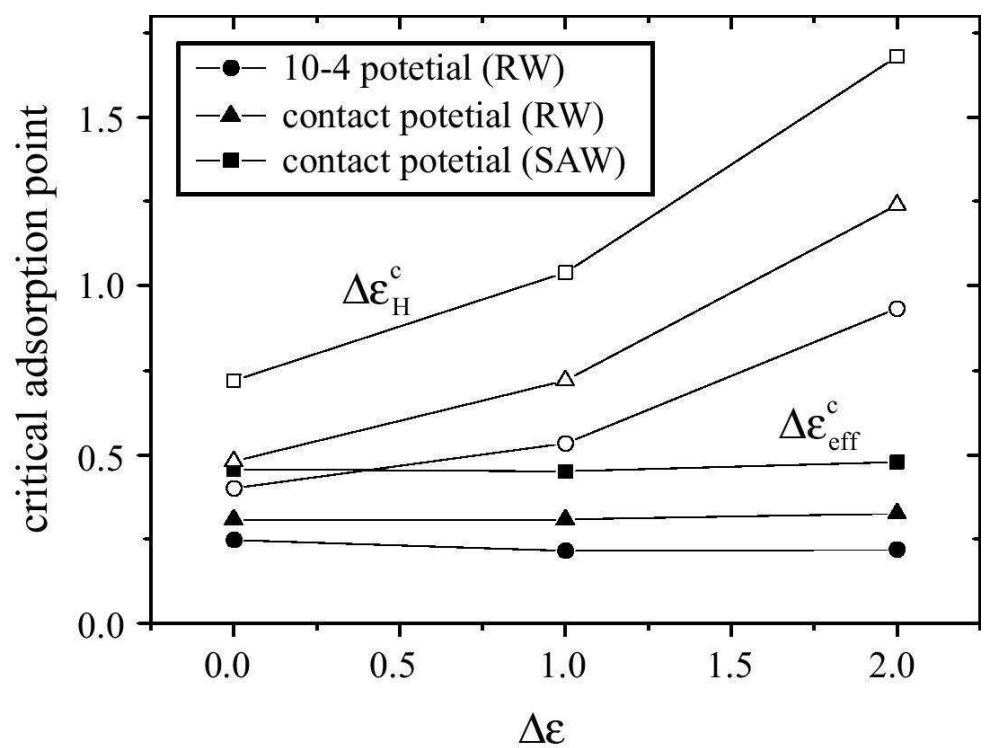

FIG. 6: $\Delta \epsilon_{H}^{c}$ and the corresponding $\epsilon_{e f f}^{c}$ (as obtained by substituting $\Delta \epsilon_{H}^{c}$ into (8) ) vs $\Delta \epsilon$. 


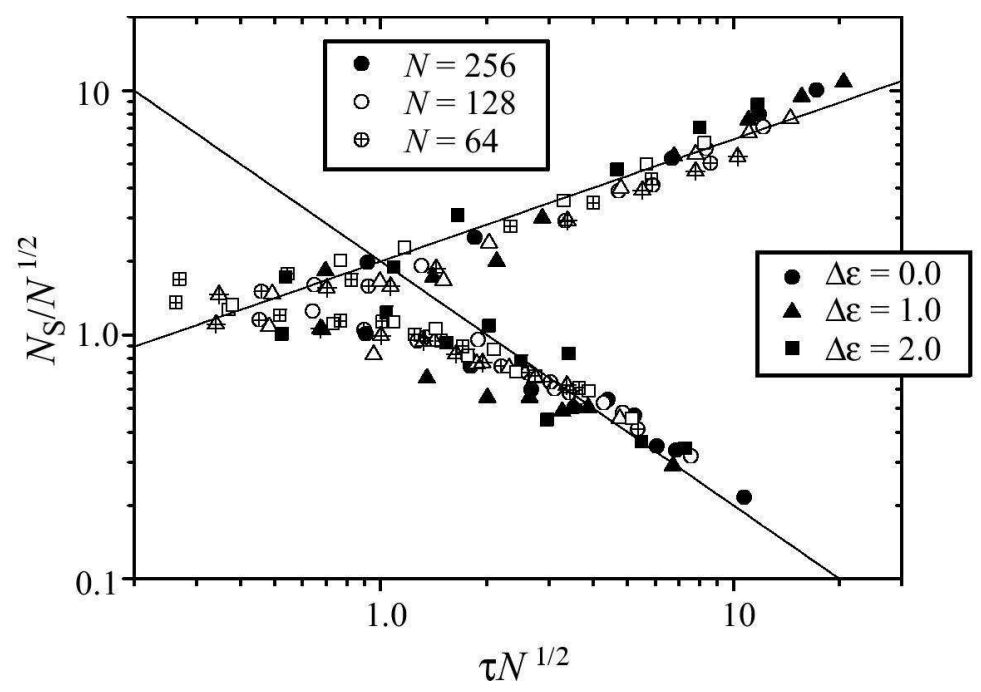

FIG. 7: $\quad N_{S} / N^{1 / 2}$ vs $\tau N^{1 / 2}$ for SAW experiencing a contact potential.

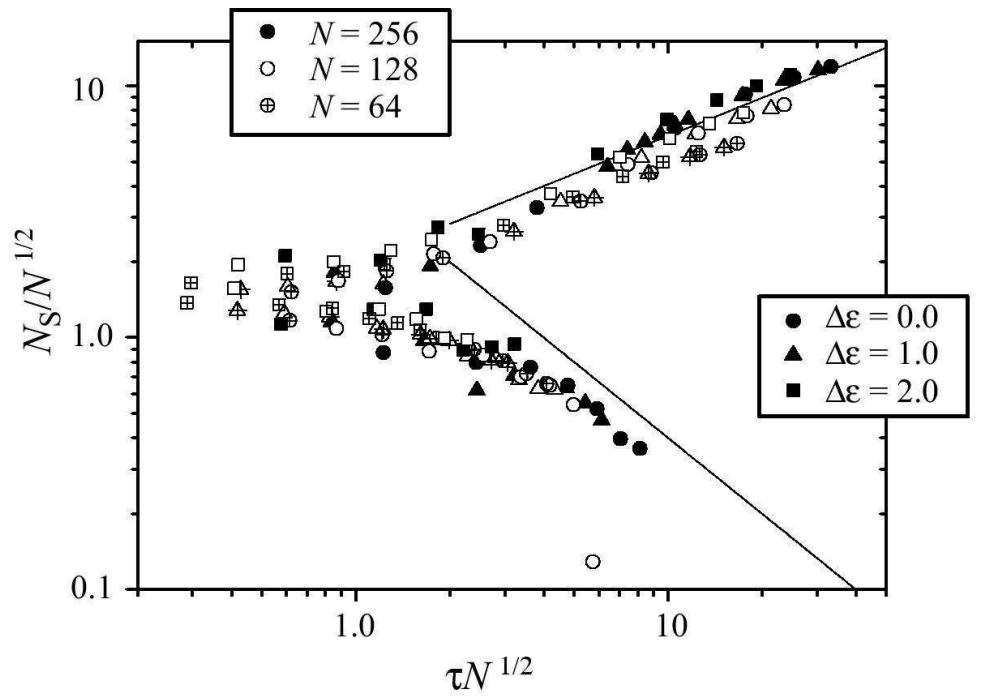

FIG. 8: $N_{S} / N^{1 / 2}$ vs $\tau N^{1 / 2}$ for RW experiencing a contact potential. 


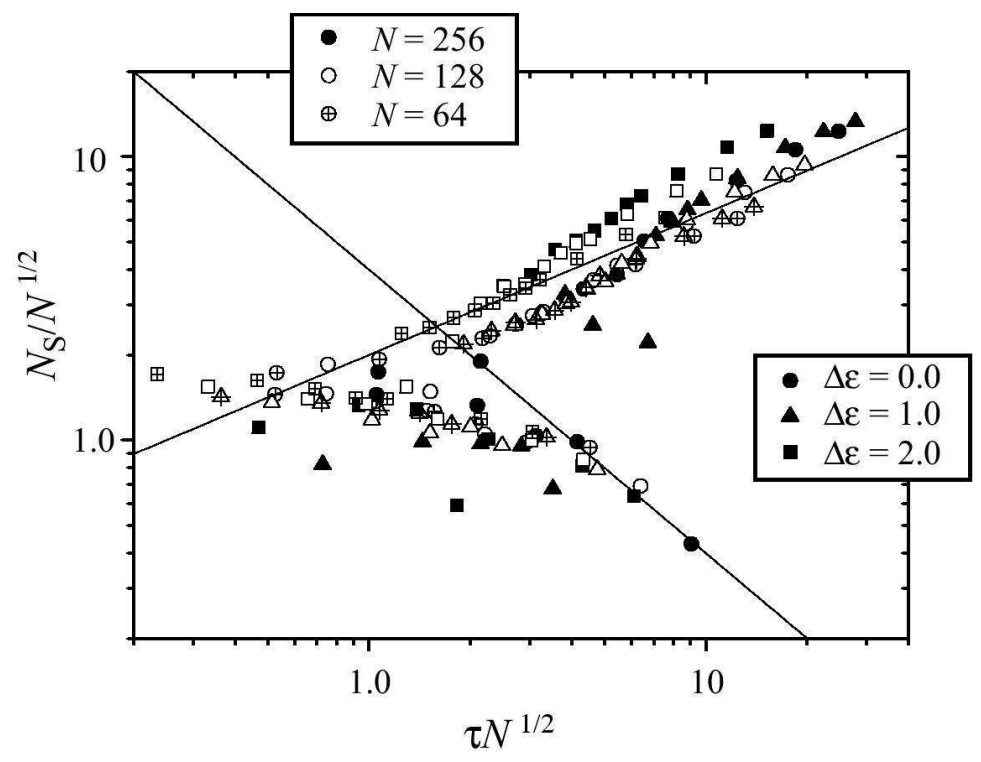

FIG. 9: $\quad N_{S} / N^{1 / 2}$ vs $\tau N^{1 / 2}$ for RW experiencing a 10-4 potential.

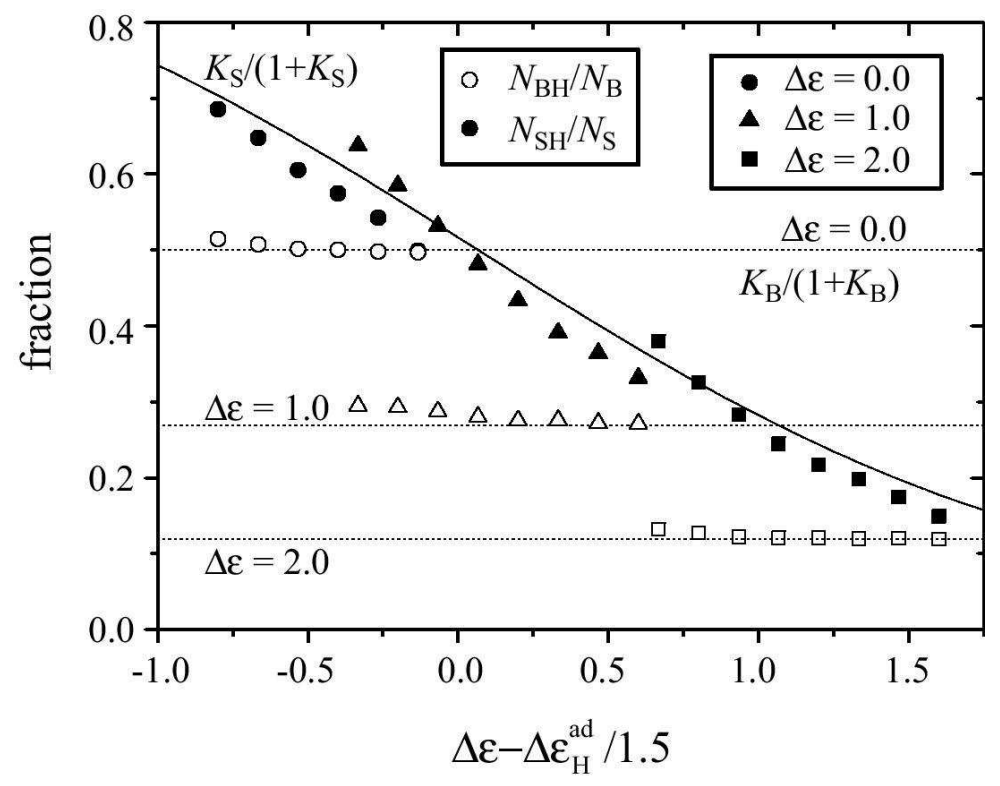

FIG. 10: $\quad N_{B H} / N_{B}$ and $N_{S H} / N_{S}$ as a function of a 10-4 potential $\Delta \epsilon-\Delta \epsilon_{H}^{a d} / 1.5$ for various $\mu_{H}^{0}-\mu_{P}^{0}=k T \Delta \epsilon$ and the corresponding curves given by (30) and (32). 